T. Kawata and I. Kubo

Nagoya Math. J.

Vol. 39 (1970), 7-21

\title{
SAMPLE PROPERTIES OF WEAKLY STATIONARY PROGESSES
}

\author{
T. KAWATA AND I. KUBO
}

1. Introduction. Let $X(t)=X(t, \omega),-\infty<t<\infty$, be a stationary stochastic process with

$$
E X(t)=0, E|X(t)|^{2}<\infty,-\infty<t<\infty
$$

and the continuous covariance function

$$
\rho(u)=\int_{-\infty}^{\infty} e^{i x u} d F(x),
$$

where $F(x)$ is the spectral distribution function. $X(t)$ then admits the harmonic representation

$$
X(t)=\int_{-\infty}^{\infty} e^{i t \lambda} d \xi(\lambda)
$$

where $\xi(\lambda)$ is a stochastic process with orthogonal increments and the property that

$$
E d \xi(\lambda)=0, \quad E|d \xi(\lambda)|^{2}=d F(\lambda) .
$$

Two stochastic processes $X(t)$ and $X_{1}(t)$ are said to be equivalent to each other, if

$$
P\left(X(t)=X_{1}(t)\right)=1, \quad \text { for each } t .
$$

When $X(t)$ is equivalent to a process continuous almost surely or differentiable almost surley, $X(t)$ is called sample continuous or sample differentiable respectively.

One of the authors has shown the following theorem [3].

Theorem A. Suppose that for a given weakly stationary process $X(t)$ there is a function $g(x)$ which is even, non-negative and non-decreasing for $x>0$ and is such that

$$
\sum_{n=1}^{\infty} \frac{1}{g(n)}<\infty
$$

Received May 28, 1969. This work partly supported by NSF Grant 9396. 
(1. 6)

$$
\int_{-\infty}^{\infty} g(x) d F(x)<\infty
$$

Then $X(t)$ is sample continuous.

The condition (1.6) with $g(x)=|x|\left(\log ^{+}|x|\right)^{\beta}, \beta>1$, implies the condition

$$
\varphi(h)=O\left(h /|\log | h \|^{r}\right) \text { for } r>2,
$$

as $h \rightarrow 0$, where $\varphi(h)=2 \rho(o)-\rho(h)-\rho(-h) \quad$ ([3] (3. 8) and (3.9)). This generalizes the Cramér-Leadbetter's result on sample continuity of a weakly stationary process ([1], p. 125).

In 2, we shall give the conditions which assure the sample differentiability of a process. We can adopt the method for the proof similar to what we did proving Theorem A, namely we make use of the approximate Fourier series [3] [6] associated with a given weakly stationary process. In 3, we shall show that the same reasoning still applies to get the "sample Hölder property".

In the paper of one of the authors [2], Theorem A was motivated by a theorem on the absolute convergence of the Fourier series of a given process truncated at $-T$ and $T$. But it involved some erroneous argument although the theorem itself is right, and the different method using the approximate Fourier series was employed to prove Theorem $A$ in [3]. In 4 , it is shown that the original way of proving is effective if some modifications are made with a slight additional condition on $g(x)$.

Finally we mention that the conditions on the existence of $g(x)$ in Theorem A are also necessary for all the weakly stationary processes with a given spectral distribution $F(x)$ to be sample continuous. This has been shown by I. Kubo [4] and will be given in a separate forthcoming paper.

\section{Sample differentiability of a weakly stationary process.}

M. Loève [5] studied the sample differentiability of a weakly stationary process and proved among others the following theorem.

THEOREM B. If the covariance function $\rho(u)$ of a weakly stationary process $X(t)$ with (1. 1$)$ and (1.2), is $(2 n+2)$-times differentiable, then $X(t)$ is sample $n$ times differentiable.

Cramér and Leadbetter [1] generalized this result to obtain Theorem $C$ below. 
Write

$$
\Delta_{u}^{2 k} \rho(-k u)=\sum_{j=0}^{2 k}(-1)^{j}\left(\begin{array}{c}
2 k \\
j
\end{array}\right) \rho((k-j) u)
$$

where $k$ is a non-negative integer.

Theorem C. If the covariance function $\rho(u)$ of a weakly stationary process $X(t)$ with (1.1) and (1.2) satisfies

$$
\Delta_{u}^{2 n+1} \rho(-k u)=O\left(|u|^{2 n+1} /|\log | u||^{q}\right), \text { as } u \rightarrow 0 \text { for } q>3,
$$

then $X(t)$ is sample n-times differentiable.

This is a slight completion of the Cramér-Leadbetter's result. They actually have shown Theorem $\mathrm{C}$ for the case $n=0,1$.

The aim of this section is to generalize Theorem $\mathrm{C}$ further.

In association with a given weakly stationary process $X(t)$ with (1. 1), (1.2) and the representation (1.3), we define a sequence of uncorrelated random variables

$$
\xi_{n}=\xi_{n}(T)=\int_{2 n \pi / T}^{2(n+1) \pi / T} d \xi(\lambda), \quad n=0, \pm 1, \cdots,
$$

where $T$ is any positive number. We also define

$$
\hat{X}(t, T)=\hat{X}(t)=\sum_{n=-\infty}^{\infty} e^{2 \pi i t / T \xi_{n}}
$$

Actually $\xi_{n}$ 's are uncorrelated because of the orthogonality of the increments of $\xi(\lambda)$ and (2.4) is well-defined, the series being interpreted to converge in $L^{2}$-norm.

However, we have shown in [3] and [4] that under the conditions either in Theorem A or in Lemma 3 below, the series in (2.4) is absolutely convergent almost surely and hence $X(t)$ may be identified to be the sum of the series. Also it was shown that in this case $\hat{X}_{k}(t)=\hat{X}\left(t, 2^{k}\right)$ converges uniformly for every finite interval $|t| \leq A$ as $k \rightarrow \infty$ almost surely to a weakly stationary process $X_{0}(t)$, which is sample continuous, and is equivalent to $X(t)$.

LEMMA 1. If

$$
\sum_{n=-\infty}^{\infty}|n|^{r}\left|\xi_{n}\right|<\infty
$$


almost surely, where $r$ is a positive integer, then $X(t)$ is equivalent to a weakly stationary process with the almost sure continuous $r$-th derivative.

Proof. Since the series on the right of (2.4) is absolutely and uniformly convergent almost surely, because of (2.5), we may suppose that $X(t)$ itself is represented by the series in (2.4) for every $t$ almost surely, and has the continuous $r$-th derivative almost surely. We shall, however, prove Lemma 1 when $r=1$. $\quad r$ repetitions of the same argument give us the required.

$$
\frac{\hat{X}(t+h)-\hat{X}(t)}{h}=\sum_{n=-\infty}^{\infty}\left[\frac{e^{2 n \pi i h / T}-1}{h} e^{2 n \pi i t / T \xi_{n}}\right] .
$$

The series on the right is dominated in absolute value by $(2 \pi / T) \sum|n|\left|\xi_{n}\right|$ almost surely and since each term converges as $h \rightarrow 0$, the limit of (2.6) as $h \rightarrow 0$ should exist and $\hat{X}^{\prime}(t)$ is given by $\frac{2 \pi i}{T} \sum_{n=-\infty}^{\infty} e^{2 n \pi i t / T} n \xi_{n}$, which is continuous almost surely. Generally $\hat{X}^{(r)}(t)$ is given by $\left(\frac{2 \pi i}{T}\right)^{r} \sum_{n=-\infty}^{\infty} e^{2 n \pi i t / T} n^{r} \xi_{n}$.

Lemma 2. Let $h(x)$ be non-negative and non-decreasing over $[0, \infty)$ and let $F(x)$ be a spectral distribution. Then the inequalities

$$
\begin{aligned}
& \frac{1}{2} \sum_{n \neq 0} h\left(\frac{|n|-1}{a}\right)(F(n+1)-F(n))^{1 / 2}+\frac{1}{2} h(0)(F(1)-F(0))^{1 / 2} \leq \\
& \leq \sum_{n} h(|n|)(F(a(n+1))-F(a n))^{1 / 2} \leq \\
& \leq\left(\frac{1}{a}+1\right)^{1 / 2} \sum_{n} h\left(\frac{|n|+1}{a}\right)(F(n+1)-F(n))^{1 / 2}
\end{aligned}
$$

hold for $0<a<1$.

Proof. Since $\sqrt{x+y} \leq \sqrt{x}+\sqrt{y}$ for $x, y \geq 0$, we have

$$
\begin{aligned}
& I= \sum_{n} h(|n|)(F(a(n+1))-F(a n))^{1 / 2} \\
&=\sum_{k}\left[h\left(\left|\left[\frac{k}{a}\right]\right|\right)\left(F\left(a\left[\frac{k}{a}\right]+a\right)-F\left(a\left[\frac{k}{a}\right]\right)\right)^{1 / 2}+\sum_{n=[k / a]+1}^{[(k+1) / a]-1} h(|n|)(F(a(n+1))-F(a n))^{1 / 2}\right] \\
& \leq \sum_{k}\left[h\left(\left|\left[\frac{k}{a}\right]\right|\right)\left(F(k)-F\left(a\left[\frac{k}{a}\right]\right)\right)^{1 / 2}+h\left(\left|\left[\frac{k}{a}\right]\right|\right)\left(F\left(a\left[\frac{k}{a}\right]+a\right)-F(k)\right)^{1 / 2}+\right. \\
&\left.+\sum_{n=[k / a]+1}^{[(k+1) / a]-1} h\left(\frac{|k|+1}{a}\right)(F(a(n+1))-F(a n))^{1 / 2}\right] \\
& \leq \sum_{k} h\left(\frac{|k|+1}{a}\right)\left(\frac{1}{a}+1\right)^{1 / 2}(F(k+1)-F(k))^{1 / 2}
\end{aligned}
$$


The last inequality is obtained by Schwarz inequality. Since $2 \sqrt{x+y} \geqq$ $\sqrt{x}+\sqrt{y}$ for $x, y \geqq 0$, we have similarly

$$
\begin{aligned}
2 I \geqq & \sum_{k}\left[h\left(\left[\frac{k+1}{a}\right]\right)\left(F(k+1)-F\left(a\left[\frac{k+1}{a}\right]\right)\right)^{1 / 2}+h\left(\left[\frac{k}{a}\right]\right)\left(F\left(a\left[\frac{k}{a}\right]+a\right)-F(k)\right)^{1 / 2}+\right. \\
& +\sum_{n=\lfloor k / a]+1}^{[(k+1) / a]-1} h\left(\frac{|k|-1}{a}\right)\left(F(a(n+1)-F(a n))^{1 / 2}\right] \geqq \\
\geqq & \sum_{k} h\left(\frac{|k|-1}{a}\right)(F(k+1)-F(k))^{1 / 2},
\end{aligned}
$$

with the agreement that $h(u)=h(0)$ for $u \leq 0$.

Lemma 3. If the spectral distribution function $F$ of a given stationary process $X(t)$ satisfies

$$
\sum_{n}|n|^{r}(F(n+1)-F(n))^{1 / 2}<\infty
$$

for a non-negative integer $r$, then (2.5) holds almost surely.

Proof. In order to show (2.5) it is sufficient to prove

$$
E \sum_{n=-\infty}^{\infty}|n|^{r}\left|\xi_{n}\right|<\infty
$$

By Lemma 2, we have that, for $0<T \leq 2 \pi$,

$$
\begin{aligned}
& E \Sigma|n|^{r}\left|\xi_{n}\right| \leq \Sigma|n|^{r}\left[E\left|\int_{2 n \pi / T}^{2(n+1) \pi / T} d \xi(\lambda)\right|^{2}\right]^{1 / 2}= \\
& \quad=\Sigma|n|^{r}\left(F\left(\frac{2(n+1) \pi}{T}\right)-F\left(\frac{2 n \pi}{T}\right)\right)^{1 / 2} \leq \\
& \quad \leq\left(\frac{T}{2 \pi}+1\right)^{1 / 2} \Sigma\left[\frac{T(|n|+1)}{2 \pi}\right]^{r}(F(n+1)-F(n))^{1 / 2}<\infty .
\end{aligned}
$$

If $T \geqq 2 \pi$, then (2.9) follows from the first inequality of (2.7).

It is easy to show that $\hat{X}^{(r)}(t)$ is a weakly stationary process, observing $\Sigma|n|^{2 r}(F(2(n+1) \pi / T)-F(2 n \pi / T))<\infty$.

Now we shall prove

THEOREM 1. If a weakly stationary process $X(t)$ with (1. 1$)$ and (1.2) satisfies (2. 8), then $X(t)$ is equivalent to a weakly stationary process which has the continuous $r$-th derivative almost surely.

Proof. First we prove the theorem for $r=1$. Denote the differential quotients of $X(t)$ and $\hat{X}(t)$ by 


$$
\begin{aligned}
& D(t, h)=\frac{X(t+h)-X(t)}{h}, \\
& \hat{D}(t, h)=\frac{\hat{X}(t+h)-\hat{X}(t)}{h}
\end{aligned}
$$

respectively. From Lemma 1, the series in (2.4) is absolutely convergent and $\hat{X}(t)$ may be supposed to be defined by this series. By Lemma 1 and Lemma 3, $\hat{X}(t)$ has the continuous derivative almost surely.

Write $\xi_{n, k}$ for $\xi_{n}$ with $T=2^{k}, \hat{X}_{k}(t)$ for the corresponding $\hat{X}(t), k$ being a positive integer. Then

$$
\begin{aligned}
\hat{X}_{k+1}(t)-\hat{X}_{k}(t)= & \sum_{n=-\infty}^{\infty} \exp \left(\frac{2 n \pi i t}{2^{k+1}}\right) \xi_{n . k+1}-\sum_{m=-\infty}^{\infty} \exp \left(\frac{2 m \pi i t}{2^{k}}\right) \xi_{m, k}= \\
= & \sum_{m=-\infty}^{\infty}\left[\exp \left(\frac{2 \pi i(2 m) t}{2^{k+1}}\right) \xi_{2 m, k+1}+\right. \\
& \left.+\exp \left(\frac{2 \pi i(2 m+1) t}{2^{k+1}}\right) \xi_{2 m+1, k+1}-\exp \left(\frac{2 m \pi i t}{2^{k}}\right) \xi_{m, k}\right] .
\end{aligned}
$$

Since $\xi_{m, k}=\xi_{2 m, k+1}+\xi_{2 m+1, k+1}$, we may write

$$
\begin{aligned}
& \hat{X}_{k+1}(t)-\hat{X}_{k}(t)= \\
& \quad=\sum_{m=-\infty}^{\infty}\left[\exp \left(\frac{2 \pi i(2 m+1) t}{2^{k+1}}\right)-\exp \left(-\frac{2 \pi i(2 m) t}{2^{k+1}}\right)\right] \xi_{2 m+1, k+1} .
\end{aligned}
$$

Write $\hat{D}_{k}(t, h)$ for the differential quotient of $\hat{X}_{k}(t)$.

Together with the relation for $\hat{X}_{k}(t+h)$ similar to (2.13) and noting that, for $|t| \leq A, A$ being a positive number,

$$
\begin{gathered}
\left|e^{i y(t+h)}-e^{i y t}-e^{i z(t+h)}+e^{i z t}\right| \leq \\
\leq\left|\left(e^{i y t}-e^{i z t}\right)\left(e^{i y h}-1\right)\right|+\left|e^{i z t}\left(e^{i y h}-e^{i z h}\right)\right| \leq \\
\leq 4\left|\sin \frac{y-z}{2} t \sin \frac{y h}{2}\right|+2\left|\sin \frac{y-z}{2} h\right| \leq|h||y-z| \cdot(1+A|y|),
\end{gathered}
$$

we obtain

(2. 15)

$$
\begin{aligned}
& \left|\hat{D}_{k+1}(t, h)-\hat{D}_{k}(t, h)\right| \leq \\
& \leq \mid \sum_{m=-\infty}^{\infty}\left[\exp \left(\frac{\pi i(2 m+1)(t+h)}{2^{k}}\right)-\exp \left(\frac{\pi i(2 m)(t+h)}{2^{k}}\right)-\right. \\
& \left.\quad-\exp \left(\frac{\pi i(2 m+1) t}{2^{k}}\right)+\exp \left(\frac{\pi i(2 m) t}{2^{k}}\right)\right] \xi_{2 m+1, k+1} \mid \leq \\
& \quad \leq \sum_{m=-\infty}^{\infty}\left(\frac{\pi}{2^{k}}+\frac{A \pi^{2}(2|m|)}{2^{k}}\right)\left|\xi_{2 m+1, k+1}\right| .
\end{aligned}
$$


Therefore we can see by Lemma 2 that for any $\varepsilon_{k}>0$

$$
\begin{aligned}
Q_{k} & \equiv P\left(\sup _{|t| \leq A, h \neq 0}\left|\hat{D}_{k+1}(t, h)-\hat{D}_{k}(t, h)\right|>\varepsilon_{k}\right) \leq \\
& \leq \frac{1}{\varepsilon_{k}} \frac{\pi}{2^{k}} \sum_{m}\left(1+\frac{2 A|m|}{2^{k}}\right) E\left|\xi_{2 m+1, k+1}\right| \leq \\
& \leq \frac{1}{\varepsilon_{k}} \frac{\pi}{2^{k}} \sum_{m}\left(1+\frac{2 A|m|}{2^{k}}\right)\left(F\left(\frac{(2 m+1) \pi}{2^{k}}\right)-F\left(\frac{2 m \pi}{2^{k}}\right)\right)^{1 / 2} \leq \\
& \leq \frac{1}{\varepsilon_{k}} \frac{\pi}{2^{k}} \sum_{n}\left(1+\frac{A|n|}{2^{k}}\right)\left(F\left(\frac{(n+1) \pi}{2^{k}}\right)-F\left(\frac{n \pi}{2^{k}}\right)\right)^{1 / 2} \leq \\
& \leq \frac{1}{\varepsilon_{k}} \frac{\left(2^{k}+\pi\right)^{1 / 2}}{2^{k} \pi^{1 / 2}} \sum_{n}(1+A(|n|+1))\left(F(n+1)-(F(n))^{1 / 2} \leq\right. \\
& \leq \frac{1}{\varepsilon_{k}} \frac{C_{1}}{2^{k / 2}}\left[\sum_{n}|n|(F(n+1)-F(n))^{1 / 2}+C_{2}(F(1)-F(0))^{1 / 2}\right],
\end{aligned}
$$

where $C_{1}$ and $C_{2}$ are constants independent of $k$. In what follows $C_{j}$, $j=3,4, \cdots$, mean some constants independent of $k$. From (2. 8), it follows that

$$
Q_{k} \leq \frac{1}{\varepsilon_{k}} \frac{C_{3}}{2^{k / 2}}
$$

If $\varepsilon_{k}$ is chosen to be $2^{-k / 4}$, then $\sum \varepsilon_{k}<\infty$ and $\sum \frac{1}{2^{k / 2} \varepsilon_{k}}<\infty$, so that $\Sigma Q_{k}<\infty$. Then Borel-Cantelli lemma gives us that, with probability one

$$
\sup _{h \neq 0,|t| \leq A}\left|\hat{D}_{k+1}(t, h)-\hat{D}_{k}(t, h)\right|<\varepsilon_{k}
$$

except for a finite number of $k$. Hence almost surely $\hat{D}_{k}(t, h)$ converges as $k \rightarrow \infty$ uniformly for $|t| \leq A$ and $h$.

Now from (2.18) we have, for $k$ larger than some $k_{0}$,

$$
\sup _{|t| \leq A}\left|\hat{D}_{k}(t, h)-\hat{D}_{m}(t, h)\right| \leq \eta_{k} \quad \text { (unifomly in } h \text { ) }
$$

almost surely, where $\eta_{k}=\sum_{j=k}^{\infty} \varepsilon_{j}$. From the italicized statement before Lemma 1 , we have, letting $m \rightarrow \infty$

$$
\left|\hat{D}_{k}(t, h)-\frac{1}{h}\left[X_{0}(t+h)-X_{0}(t)\right]\right| \leq \eta_{k} \quad \text { (uniformly in } h \text { ) }
$$

almost surely. Let $h \rightarrow 0$. Then from Lemma 1 and 2 with $r=1, \hat{D}_{k}(t, h)$ converges almost surely and hence $X_{0}(t)$ is differentiable almost surely, and is equivalent to $X(t)$. 
Finally (2. 19) implies that the derivative $\hat{X}_{k}^{\prime}(t)$ of $\hat{X}_{k}(t)$ converges uniformly to the derivative $X_{0}^{\prime}(t)$ of $X_{0}(t)$. Since Lemmas 1 and 2 give us that $\hat{X}^{\prime}{ }_{k}(t)$ is continuous almost surely, $X^{\prime}{ }_{0}(t)$ is also sample continuous for every $|t| \leq A$. This proves the theorem for the case $r=1$.

Repeating similar arguments, the general case is shown.

THEOREM 2. If, for a given weakly stationary process $X(t)$ with (1. 1) and (1. 2), there is a function $g(x),-\infty<x<\infty$, which is non-negative, even and nondecreasing for $x \geqq 0$ and satisfies

$$
\sum_{n=1}^{\infty} \frac{n^{2 r}}{g(n)}<\infty
$$

$$
\int g(x) d F(x)<\infty
$$

then $X(t)$ is equivalent to a weakly stationary process which has the continuous $r$-th derivative almost surely.

Proof. By Schwarz inequality,

$$
\begin{aligned}
{\left[\sum_{n=0}^{\infty}|n|^{r}(F(n+1)-F(n))^{1 / 2}\right]^{2} } & \leq \sum_{n=0}^{\infty}-\frac{n^{2 r}}{g(n)} \sum_{n=0}^{\infty} g(n)(F(n+1)-F(n)) \\
& \leq \sum_{n=0}^{\infty} \frac{n^{2 r}}{g(n)} \int_{0}^{\infty} g(x) d F(x)<\infty
\end{aligned}
$$

Similarly, we have $\sum_{n=-1}^{-\infty}|n|^{r}(F(n+1)-F(n))^{1 / 2}<\infty$. By Theorem 1, the proof is completed.

ExAmple 1. If, for some $\varepsilon>0$ and $B>0$

$$
\int_{B<|x|}|x|^{2 r+1} \log |x| \cdot \log _{(2)}|x| \cdot \cdots \log _{(n)}|x| \cdot\left(\log _{(n+1)}|x|\right)^{1+\varepsilon} d F(x)<\infty
$$

holds, then $X(t)$ is sample $r$-times differentiable, where $\log _{(1)} x=\log x$ and $\log _{(n+1)} x=\log \left(\log _{(n)} x\right)$ for $n \geqq 1$.

ExAmple 2. Suppose that $F(x)$ is absolutely continuous with the density $f(x)$. If

$$
|f(x)| \leq\left[|x|^{r+1} \log |x| \cdot \log _{(2)}|x| \cdots \log _{(n)}|x| \cdot\left(\log _{(n+1)}|x|\right)^{1+\varepsilon}\right]^{-2} .
$$

holds for sufficiently large $|x|$ with some $\varepsilon>0$, then $X(t)$ is sample $r$-times differentiable. 
Example 3. Besides the same assumption in Example 2, further suppose that $f(x)$ is non-decreasing as $x \rightarrow \pm \infty$. If

$$
\int|x|^{r} f^{1 / 2}(x) d x<\infty
$$

holds, then $X(t)$ is sample $r$-times differentiable.

\section{Sample Hölder continuity.}

Let $\Psi(h)$ be a non-decreasing function defined over an interval $(0,1]$ such that $\Psi(h)$ decreases to zero as $h$ does. If a function $f(x)$ on $(a, b)$ satisfies

$$
|f(t+h)-f(t)| \leq M \Psi(h)
$$

for $t, t+h \in(a, b),|h|<1$ with some $M$, then it is said to be $\Psi$-Hölder continuous.

We are going to give sufficient conditions which assure the sample $\Psi$ Hölder continuity of a weakly stationary process. The method similar to the one applied to the proofs of Theorems 1 and 2 is also applicable.

Lemma 4. Let $\Psi(h)$ be a non-decreasing function over $(0,1]$ such that $\Psi(h) / h$ is non-increasing. Then for $0<h \leq 1$

$$
\begin{aligned}
& |\sin x h| \leq \Psi(h) / \Psi\left(x^{-1}\right), \quad \text { for } \quad x \geqq 1, \\
& |\sin x h| \leq \Psi(h) / \Psi\left(\frac{1}{x+1}\right), \quad \text { for } \quad x \geqq 0 .
\end{aligned}
$$

Proof. If $0<x h<1$, then

$$
|\sin x h| \leq x h=\frac{\Psi(h)}{\Psi\left(x^{-1}\right)} \cdot \frac{\Psi\left(x^{-1}\right)}{x^{-1}} \cdot \frac{h}{\Psi(h)} \leq \frac{\Psi(h)}{\Psi\left(x^{-1}\right)} .
$$

If $x h \geqq 1$, then since $\Psi(h)$ is non-decreasing

$$
|\sin x h| \leq 1 \leq \Psi(h) / \Psi\left(x^{-1}\right) .
$$

Similarly, we can prove (3.3) observing $x h \leqslant h(x+1)$.

Lemma 5. If $\Psi(h)$ is non-decreasing and $\Psi(h) / h$ is non-increasing over $(0,1]$, and

$$
\sum_{n \neq 0} \Psi^{-1}\left(\frac{1}{|n|}\right)\left|\xi_{n}\right|<\infty
$$


almost surely, then $\hat{X}(t)=X(t, T), T>\pi$, is sample $\Psi$-Hölder continuous where $\xi_{n}$ is defined by (2. 3) and $\hat{X}(t)$ is defined by (2.4).*

Proof. Using Lemma 4, we have

$$
\begin{aligned}
& |X(t+h)-X(t)| \leq \sum_{n \neq 0}|\sin n h \pi / T|\left|\xi_{n}\right| \leq \\
& \leq \Psi\left(\left|\frac{h \pi}{T}\right|\right) \sum_{n \neq 0} \Psi^{-1}\left(\frac{1}{|n|}\right) \quad\left|\xi_{n}\right| \leq \Psi(|h|) \sum_{n \neq 0} \Psi^{-1}\left(\frac{1}{|n|}\right)\left|\xi_{n}\right| .
\end{aligned}
$$

Lemma 6. If the spectral distribution function $F(x)$ satisfies

$$
\sum_{n \neq 0} \Psi^{-1}\left(\frac{1}{|n|}\right)(F(n+1)-F(n))^{1 / 2}<\infty .
$$

Then (3. 4) holds almost surely, where $\Psi(h)$ is the function in Lemma 4. Hence $X(t)$ is $\Psi$-Hölder continuous almost surely.

The proof is carried out as in that of Lemma 3.

Theorem 3. If a given weakly stationary process $X(t)$ satisfies (3. 6), then $X(t)$ is equivalent to a weakly stationary process which is $\Psi$-Hölder continuous almost surely, where $\Psi(h)$ is the function in Lemma 4.

The proof is very similar to that for Theorem 1. Write

$$
D_{\Psi}(t, h)=\frac{X(t+h)-X(t)}{\Psi(|h|)}, \hat{D}_{\Psi, k}(t, h)=\frac{\hat{X}_{k}(t+h)-\hat{X}_{k}(t)}{\Psi(|h|)},
$$

where $\hat{X}_{k}(t)$ is, as before, defined by (2.4) with $T=2^{k}$. Using the same notations as in the proof of Theorem 1, we have, analogously to (2. 15), by Lemma 4 and (2. 14),

$$
\begin{aligned}
& \left|\hat{D}_{\Psi, k+1}(t, h)-\hat{D}_{\Psi, k}(t, h)\right| \leq \\
& \quad \leq \frac{1}{\Psi(|h|)} \sum_{m=-\infty}^{\infty}\left(4\left|\sin \frac{\pi t}{2^{k+1}} \sin \frac{2 m \pi h}{2^{k+1}}\right|+2\left|\sin \frac{\pi h}{2^{k+1}}\right|\right)\left|\xi_{2 m+1, k+1}\right| \\
& \quad \leq \sum_{m=-\infty}^{\infty}\left(\frac{\pi}{2^{k}} \Psi^{-1}(1)+\frac{2 \pi A}{2^{k}} \Psi^{-1}\left(\frac{2^{k+1}}{2 \pi|m|+2^{k+1}}\right)\right)\left|\xi_{2 m+1, k+1}\right|,
\end{aligned}
$$

for $|t| \leq A$. Therefore we obtain by Lemma 2

$$
\begin{aligned}
Q^{\prime}{ }_{k} & \equiv P\left(\sup _{0<|h| \leq 1,|t| \leq A}\left|\hat{D}_{\Psi_{, k+1}}(t, h)-\hat{D}_{\Psi_{, k}}(t, h)\right|>\varepsilon_{k}\right) \leq \\
& \leq \frac{1}{\varepsilon_{k}} \frac{\pi}{2^{k}} \sum_{m=-\infty}^{\infty}\left(\Psi^{-1}(1)+2 A \Psi^{-1}\left(\frac{2^{k+1}}{2 \pi|m|+2^{k+1}}\right)\right)\left(F\left(\frac{(2 m+1) \pi}{2^{k}}\right)-F\left(\frac{2 m \pi}{2^{k}}\right)\right)^{1 / 2} \\
& * \Psi^{-n}(x)=(\Psi(x))^{-n} .
\end{aligned}
$$




$$
\begin{aligned}
& \leq \frac{1}{\varepsilon_{k}} \frac{\pi}{2^{k}} \sum_{n=-\infty}^{\infty}\left(\Psi^{-1}(1)+2 A \Psi^{-1}\left(\frac{2^{k+1}}{\pi|n|+2^{k+1}}\right)\right)\left(F\left(\frac{(n+1) \pi}{2^{k}}\right)-F\left(\frac{n \pi}{2^{k}}\right)\right)^{1 / 2} \leq \\
& \leq \frac{1}{\varepsilon_{k}} \frac{\pi}{2^{k}} \sqrt{\frac{2^{k}}{\pi}+1} \sum_{n=-\infty}^{\infty}\left(\Psi^{-1}(1)+2 A \Psi^{-1}\left(\frac{2}{|n|+3}\right)\right)(F(n+1)-F(n))^{1 / 2} .
\end{aligned}
$$

Since $\Psi^{-1}\left(\frac{2}{|n|+3}\right) \leq \frac{(|n|+3)}{|n|} \Psi^{-1}\left(\frac{1}{|n|}\right)$ and $\Psi\left(\frac{1}{|n|}\right) \leq \Psi(1)$ for $n \neq 0$, we get

$$
\begin{aligned}
Q^{\prime}{ }_{k} & \leq \frac{1}{\varepsilon_{k}} \frac{C_{4}}{2^{k / 2}}\left[(F(1)-F(0))^{1 / 2}+\sum_{n \neq 0} \Psi^{-1}\left(\frac{1}{|n|}\right)(F(n+1)-F(n))^{1 / 2}\right] \leq \\
& \leq \frac{1}{\varepsilon_{k}} \frac{C_{5}}{2^{k / 2}} .
\end{aligned}
$$

Choosing $\varepsilon_{k}$ as in the proof of Theorem 1, we see from (3. 7) that $\hat{X}_{k}(t)$ converges uniformly to a weakly stationary process $X_{0}(t)$ and

$$
\left|\hat{D}_{\Psi, k}(t, h)-\frac{\hat{X}_{0}(t+h)-\hat{X}_{0}(t)}{\Psi(|h|)}\right| \leq \varepsilon_{k} \quad \text { for } \quad k \geqq k_{0} \text {. }
$$

By Lemma 5, $\sup _{0<|h| \leq 1,|t| \leq A}\left|\hat{D}_{\mathscr{T}, k}(t, h)\right|<\infty$ almost surely, we conclude that $X_{0}(t)$ is $\Psi$-Hölder continuous for $|t| \leq A$ for any $A>0$, which completes the proof.

THeOREM 4. If for a given weakly stationary process $X(t)$, there is an even, non-negative, non-decreasing function $g(x)$ such that

$$
\begin{aligned}
& \sum_{n=1}^{\infty} \Psi^{-2}\left(\frac{1}{n}\right) \cdot g^{-1}(n)<\infty \\
& \int g(x) d F(x)<\infty
\end{aligned}
$$

Then $X(t)$ is equivalent to a weakly stationary process which is $\Psi$-Hölder continuous almost surely, where $\Psi(h)$ is the function in Lemma 4.

Proof. By (3. 8) and (3. 9), we have

$$
\begin{gathered}
{\left[\sum_{n=1}^{\infty} \Psi^{-1}\left(\frac{1}{n}\right)(F(n+1)-F(n))^{1 / 2}\right]^{2} \leq \sum_{n=1}^{\infty} \Psi^{-2}\left(\frac{1}{n}\right) g^{-1}(n) \cdot \sum_{n=1}^{\infty} g(n)(F(n+1)-F(n))} \\
\leq \sum_{n=1}^{\infty} \Psi^{-2}\left(\frac{1}{n}\right) g^{-1}(n) \cdot \int_{1}^{\infty} g(x) d F(x)<\infty .
\end{gathered}
$$


Similarly, we can see that $\sum_{n=-1}^{-\infty} \Psi^{-1}\left(\frac{1}{|n|}\right)(F(n+1)-F(n))^{1 / 2}<\infty$. Hence the assertion follows from Theorem 3.

Example 4. Suppose that $F(x)$ is absolutely continuous with the density $f(x)$ and that $f(x)$ is non-increasing as $x \rightarrow \pm \infty$. If

$$
\int_{|x|>1} \Psi^{-1}\left(\frac{1}{|x|}\right) f^{1 / 2}(x) d x<\infty
$$

then $X(t)$ is sample $\Psi$-Hölder continuous.

EXAmple 5. If a separable stationary process $X(t)$ satisfies

$$
\int_{|x| \geq B} \Psi^{-2}\left(\frac{1}{|x|}\right)|x| \cdot \log |x| \cdot \log _{(2)}|x| \cdots \log _{(n)}|x| \cdot\left(\log _{(n+1)}|x|\right)^{1+\varepsilon} d F(x)<\infty,
$$

for sufficiently large $B>0$ with $\varepsilon>0$, then

$$
\lim _{h \rightarrow 0} \sup _{|t| \leq A} \frac{|X(t+h)-X(t)|}{\Psi(h)}=0 \quad \text { a.s.. }
$$

Especially if, $F(x)$ is absolutely continuous with the density $f(x)$ which satisfies

$$
|f(x)| \leq \Psi^{2}\left(\frac{1}{|x|}\right)\left[|x| \cdot \log |x| \cdots \log _{(n)}|x| \cdot\left(\log _{(n+1)}|x|\right)^{1+\varepsilon}\right]^{-2},
$$

then (3. 11) holds.

\section{Absolute convergence of the Fourier series of a weakly stationary process.}

Let $X(t)$ be a weakly stationary process described in 1 . Let $T$ be any positive number. Define

$$
\begin{aligned}
Y(t)=X(t), & t \geq 0, \\
X(-t), & t \leq 0 .
\end{aligned}
$$

We consider the Fourier series of $Y(t)$ over $(-T, T)$,

$$
\begin{aligned}
& A_{n}=\frac{1}{T} \int_{-T}^{T} Y(t) \cos \frac{n \pi t}{T} d t, \\
& \frac{1}{2} A_{0}+\sum_{n=1}^{\infty} A_{n} \cos \frac{n \pi t}{T} .
\end{aligned}
$$

As in [2], 
(4. 4) $E A_{n} \bar{A}_{m}=8 e^{i \pi(n-m) / 2} \int_{-\infty}^{\infty} \frac{\sin \frac{\lambda T+\pi n}{2} \sin \frac{\lambda T+\pi m}{2} \cdot \lambda^{2} T^{2}}{\left(\lambda^{2} T^{2}-n^{2} \pi^{2}\right)\left(\lambda^{2} T^{2}-m^{2} \pi^{2}\right)} d F(\lambda)$,

$$
E\left|A_{n}\right|^{2}=8 \int_{-\infty}^{\infty} \frac{\sin ^{2}\left(\frac{\lambda T+n \pi}{2}\right) \cdot \lambda^{2} T^{2}}{\left(\lambda^{2} T^{2}-n^{2} \pi^{2}\right)^{2}} d F(\lambda) \text {. }
$$

Theorem 5. Let $g(x)$ be even, non-negative and non-decreasing for $x>0$, such that $g(x) / x^{2}$ is non-increasing for large $x$ and

$$
\sum_{n=1}^{\infty} \frac{1}{g(n)}<\infty
$$

If

$$
\int_{-\infty}^{\infty} g(x) d F(x)<\infty
$$

then $\sum_{0}^{\infty}\left|A_{n}\right|$ converges almost surely.

Proof. We may suppose that $g(x) / x^{2}$ is non-decreasing over $(0, \infty)$. In fact, if $g(x) / x^{2}$ is non-increasing for $x \geqq B$, then we may define $g(x)$ as it is for $(x \geqq B)$, and $g(B)(x / B)^{2}$ for $(x \leq B)$. By (4. 5),

$$
\begin{aligned}
\sum_{n=2}^{\infty} g(n) E\left|A_{n}\right|^{2}= & 8 \int_{-\infty}^{\infty} \sum_{n=2}^{\infty} g(n) \frac{\sin ^{2}\left[\frac{1}{2}(|\lambda| T-n \pi)\right] \lambda^{2} T^{2}}{(|\lambda| T+\pi n)^{2}(|\lambda| T-\pi n)^{2}} d F(\lambda)= \\
= & 8 \int_{|\lambda|>\pi / T}\left(\sum_{n \geq[|\lambda| T / \pi]+2}\right) d F(\lambda)+8 \int_{|\lambda|>\pi / T}\left(\sum_{n=[|\lambda| T / T / \pi]+1}^{|| \lambda]}\right) d F(\lambda) \\
& +8 \int_{|\lambda|>2 \pi / T}\left(\sum_{[|\lambda| T / \pi \mid-1 \geq n}\right) d F(\lambda)+8 \int_{|\lambda| \leq \pi / T}\left(\sum_{n=2}^{\infty}\right) d F(\lambda)= \\
= & I_{1}+I_{2}+I_{3}+I_{4},
\end{aligned}
$$

say.

Noting that

$$
g(A x) \leq A^{2} g(x),
$$

for $A>1 x \geqq 1$ which, follows from the assumption that $g(x) / x^{2}$ is nonincreasing for $x \geqq 1$, we see that 


$$
\begin{aligned}
I_{1} & \leq 8 \int_{|\lambda|>\pi / T} n \geq \mid \sum_{|\lambda| T / \pi]+2} \frac{g(n) \lambda^{2} T^{2}}{\pi^{2}\left(n-\frac{|\lambda| T}{\pi}-1\right)^{2}(|\lambda| T+\pi n)^{2}} d F(\lambda) \leq \\
& \leq 8 C_{1} \int_{|\lambda| \geq \pi / T} g\left(\frac{\lambda T}{\pi}\right) d F(\lambda)<C_{2} T^{2} \int_{|\lambda| \geq \pi / T} g(\lambda) d F(\lambda)<\infty,
\end{aligned}
$$

where $C_{1}$ and $C_{2}$ are constants. Here we have used that

$$
\begin{aligned}
& g(n) \lambda^{2} T^{2} /(|\lambda| T+\pi n)^{2} \leq g(\lambda T / \pi) . \\
& I_{2} \leq C_{3} \int_{|\lambda|>\pi / T}\left[g\left(\frac{\lambda T}{\pi}+1\right)+g\left(\frac{\lambda T}{\pi}\right)\right] d F(\lambda) \leq C_{4} T^{2} \int_{|\lambda|>\pi / T} g(\lambda) d F(\lambda)<\infty,
\end{aligned}
$$

$C_{3}, C_{4}$ being constants.

$$
\begin{aligned}
I_{3} & \leq \int_{|\lambda|>\pi / T} \sum_{n \leq[\lambda T / \pi]-1} \frac{\lambda^{2} T^{2} g([\lambda T / \pi]-1)}{(|\lambda| T-\pi n)^{2}(|\lambda| T+2)^{2}} d F(\lambda) \leq \\
& \leq C_{5} \sum_{m=1}^{\infty} \frac{1}{m^{2}} \int_{|\lambda|>\pi / T} g\left(\frac{\lambda T}{\pi}\right) d F(\lambda) \leq C_{6} T^{2} \int_{|\lambda| \geq \pi / T} g(\lambda) d F(\lambda)<\infty,
\end{aligned}
$$

where $C_{5}$ and $C_{6}$ are constants.

Since $\lambda^{2} T^{2}(|\lambda| T-\pi n)^{2} \leq(n-1)^{-2}$ for $|\lambda| T \leq \pi$,

$$
I_{4} \leq 8 \sum_{n=2}^{\infty} \frac{g(n)}{\pi^{2} n^{2}(n-1)^{2}} \int_{|\lambda| \leq \pi / T} d F(\lambda) .
$$

Since $g(n) \leq n^{2} g(1)$ from (4. 8),

$$
I_{4}<\infty
$$

Hence we have obtained that

$$
\sum_{n=2}^{\infty} g(n) E\left|A_{n}\right|^{2}<\infty
$$

From this, our conclusion follows immediately, for

$$
\begin{aligned}
E \sum_{n=2}^{\infty}\left|A_{n}\right| & =E \sum_{n=2}^{\infty} \frac{1}{g^{1 / 2}(n)} g^{1 / 2}(n)\left|A_{n}\right| \leq \\
& \leq\left[\sum_{n=2}^{\infty} \frac{1}{g(n)}\right]^{1 / 2} E\left[\sum_{n=2}^{\infty} g(n)\left|A_{n}\right|^{2}\right]^{1 / 2} \leq \\
& \leq\left[\sum_{n=2}^{\infty} \frac{1}{g(n)}\left[E \sum_{n=2}^{\infty} g(n)\left|A_{n}\right|^{2}\right]^{1 / 2}\right.
\end{aligned}
$$


which is finite by (4.6) and (4.8), and $E \Sigma\left|A_{n}\right|<\infty$ implies the almost sure convergence of $\Sigma\left|A_{n}\right|$.

As an implication of the conclusion of Theorem 5 is that $X(t)$ is sample continuous in $(0, T)$ for every $T>0$ which, of course, implies that $X(t)$ is sample continuous in $(0, \infty)$. However, for this statement we need the unnecessary condition that $g(x) x^{2}$ is non-decreasing.

\section{REFERENCES}

[ 1 ] H. Cramér-M.R. Leadbetter, Stationary and related stochastic processes. John Wiley, New York, 1967.

[2] T. Kawata, On the Fourier series of a stationary process. Z. Wahrsch. 6 (1966) 224-245.

[ 3 ] T. Kawata, On the Fourier series of a stationary process, II. Z. Wahrsch. 13 (1969) 25-38.

[4] I. Kubo, On a necessary condition for the sample path continuity of weakly stationary processes. Nagoya Math. J. 38 (1970) 103-111.

[ 5 ] M. Loéve, Supplement to P. Lévy, Processus stochastique et mouvement brownien. GauthierVillars, Paris, 1948.

[6] A. Papoulis, Probability, random variables and stochastic processes. McGraw-Hill, New York, 1965.

The Catholic University of America, and

Nagoya University 